\title{
Some Characterizations of Open, Closed, and Continuous Mappings
}

\author{
Navpreet Singh Noorie and Rajni Bala \\ Department of Mathematics, Faculty of Physical Sciences, Punjabi University, Patiala 147002, India
}

Correspondence should be addressed to Navpreet Singh Noorie, navresmath@yahoo.com

Received 9 October 2007; Accepted 19 January 2008

Recommended by Sehie Park

\begin{abstract}
We obtain new characterizations of open maps in terms of closures, of closed maps in terms of interiors, and of continuous maps in terms of interiors. Further open (closed) onto maps $f: X \rightarrow Y$ are described in terms of images under $f$ of certain closed (open) sets in X. Continuity of (onto) maps is also characterized in terms of saturated sets.

Copyright (C) 2008 N. S. Noorie and R. Bala. This is an open access article distributed under the Creative Commons Attribution License, which permits unrestricted use, distribution, and reproduction in any medium, provided the original work is properly cited.
\end{abstract}

\section{Introduction}

It is well known that a map $f: X \rightarrow Y$ between arbitrary topologicalspaces is open if and only if $(f(A))^{O} \supset f\left(A^{O}\right)$ for arbitrarysubsets $A$ of $X$, where $A^{O}$ denotes the interior of $A$ (see [1]). On the other hand, Schochetman has shown in [2] that if $X$ and $Y$ are metric spaces, then $f$ is open if and only if $f^{-1}(y) \subset \limsup _{n} f^{-1}\left(y_{n}\right)$, for every convergent sequence $\left\{y_{n}\right\}$ with $\lim _{n} y_{n}=y$ in $Y$. We are motivated by the simple but important observation that not only is the $\operatorname{set}_{\limsup } f^{-1}\left(y_{n}\right)$ closed (special case of [2, Lemma 2.1]) but the set $\left\{y \in Y: f^{-1}(y) \subset \limsup _{n} f^{-1}\left(y_{n}\right)\right\}$ is also closed whenever $f$ is an open map. It is then easy to generalize that a map $f: X \rightarrow Y$ between arbitrary topological spaces is open if and only if for any closed set $F$ in $X$ the set $f^{\#}(F)=\left\{y \in Y: f^{-1}(y) \subset F\right\}$ is closed in $Y$. This raises the question if we can describe open maps in terms of inclusion relation involving closures of certain subsets, perhaps involving $f^{\#}$, rather than in terms of the well-known inclusion relation involving interiors mentioned in the beginning.

Further, it has been shown in [3, 2.5 Problem 321] that an onto map $f: X \rightarrow Y$ is closed if and only if for every open set $U$ in $X$, the set $f^{\#}(U)=\left\{y \in Y: f^{-1}(y) \subset U\right\}$ is open in $Y$. (We see in Theorem 2.10 below that we can drop the condition of onto in this result.) This again suggests the possibility of describing closed maps in terms of inclusion relation involving interiors of certain subsets, perhaps involving $f^{\#}$, rather than in terms of the well-known inclusion relation involving closures, namely, that a map $f: X \rightarrow Y$ between 
arbitrary topological spaces is closed if and only if $\mathrm{Cl}(f(A)) \subset f(\mathrm{Cl}(A))$ for arbitrary subsets $A$ of $X$, where $\mathrm{Cl}(A)$ denotes the closure of $A$ (see [1]).

In this paper,we obtain new characterizations of open maps in terms of closures (Theorem 2.7 below), closed maps in terms of interiors (Theorem 2.10 below), and continuous maps in terms of interiors (Theorem 2.13 below). Further open (closed) onto maps $f: X \rightarrow Y$ are described in terms of images under $f$ of certain closed (open) sets in $X$ in Corollary 2.8 (Corollary 2.11). Continuity of (onto) maps is also characterized in terms of saturated sets in Corollary 2.15 (Theorem 2.14).

Notation 1 . For any sets $X$ and $Y$, let $f: X \rightarrow Y$ be any map and $E$ any subset of $X$. Then

(i) $f^{\#}(E)=\left\{y \in Y: f^{-1}(y) \subset E\right\}$;

(ii) $E^{\#}=f^{-1}\left(f^{\#}(E)\right)$.

Remark 1.1. Let $f: X \rightarrow Y$ be continuous and $E$ be any subset of $X$, where $X$ and $Y$ are topological spaces. Then $E^{\#}$ is open in $X$ whenever $f^{\#}(E)$ is open in $Y$. The converse holds, if $f$ is onto and follows from Theorem 2.14 below.

\section{Results}

Definition 2.1. For any sets $X$ and $Y$, a subset $E$ of $X$ will be called a saturated subset of $X$ under a map $f: X \rightarrow Y$ if for some subset $B$ of $Y, E=f^{-1}(B)$. Equivalently, if $E=f^{-1}(f(E)$ ).

Remark 2.2. For any map $f: X \rightarrow Y, E^{\#}$ is saturated for each subset $E$ of $X$.

We begin with the following lemmas. The proof of the Lemma 2.3 is straightforward from the definitions and is omitted.

Lemma 2.3. For any sets $X$ and $Y$, let $f: X \rightarrow Y$ be any map and $E$ be any subset of $X$. Then

(i) if $A \subset B$, then $f^{\#}(A) \subset f^{\#}(B)$;

(ii) $f\left(E^{\#}\right)=f^{\#}(E) \cap f(X)$;

(iii) $E^{\#}=\left\{f^{-1} y: y \in Y\right.$ and $\left.f^{-1} y \subset E\right\} \subset E$;

(iv) $f^{\#}(\phi)=[f(X)]^{C}$;

(v) $f^{\#}(X)=Y, X^{\#}=X$, and $\phi^{\#}=\phi$;

(vi) $f\left(E^{\#}\right)=f^{\#}(E) \cap f(E)$;

(vii) $f^{\#}\left(E^{C}\right)=[f(E)]^{C}$ and so $f^{\#}(E)=\left[f\left(E^{C}\right)\right]^{C}$ and $f(E)=\left[f^{\#}\left(E^{C}\right)\right]^{C}$;

(viii) $f^{\#}\left(E^{\#}\right)=f^{\#}(E)$;

(ix) $E$ is saturated if and only if $E=E^{\#}$.

Remark 2.4. If $f: X \rightarrow Y$ is continuous, then for any saturated set $E$ in $X, E$ is open in $X$ whenever $f^{\#}(E)$ is open in $Y$. This follows from Remark 1.1 and (ix) above.

The following lemma gives characterization of onto maps. 
Lemma 2.5. For any map $f: X \rightarrow Y$, where $X$ and $Y$ are any sets, the following conditions are equivalent:
(a) $f$ is onto;
(b) $f^{\#}(A)=f\left(A^{\#}\right)$ for each subset $A$ of $X$;
(c) $f^{\#}(A) \subset f(A)$ for each subset $A$ of $X$;
(d) $f^{\#}(\phi)=\phi$.

Proof. (a) $\Rightarrow$ (b) follows from the definition of $A^{\#}$.

(b) $\Rightarrow$ (c) follows from Lemma 2.3(iii).

$(\mathrm{c}) \Rightarrow(\mathrm{d})$ is obvious.

(d) $\Rightarrow$ (a) follows from Lemma 2.3(iv).

Remark 2.6. From now onwards, all spaces are assumed to be arbitrary topological spaces.

Theorem 2.7. For any map $f: X \rightarrow Y$, the following conditions are equivalent.

(a) $f$ is an open map, that is, for each subset $A$ of $X, f\left(A^{O}\right) \subset[f(A)]^{O}$;

(b) for each subset $A$ of $X, \mathrm{Cl}\left(f^{\#}(A)\right) \subset f^{\#}(\mathrm{Cl}(A))$;

(c) for each closed subset $F$ of $X, f^{\#}(F)$ is closed in $Y$.

Proof. (a) $\Rightarrow$ (b): By Lemma 2.3(vii), $\mathrm{Cl}\left(f^{\#}(A)\right)=\mathrm{Cl}\left(\left(f\left(A^{C}\right)\right)^{C}\right)=\left[\left(f\left(A^{C}\right)\right)^{O}\right]^{C} \subset\left[f\left(\left(A^{C}\right)^{O}\right)\right]^{C}$, since (a) holds. Therefore, $\mathrm{Cl}\left[f^{\#}(A)\right] \subset\left[f\left(\left(A^{C}\right)^{O}\right)\right]^{C}=\left[f\left((\mathrm{Cl}(A))^{C}\right)\right]^{C}=f^{\#}(\mathrm{Cl}(A))$ by Lemma 2.3(vii) again. Hence, $\mathrm{Cl}\left[f^{\#}(A)\right] \subset f^{\#}(\mathrm{Cl}(A))$ and so (b) holds.

$(\mathrm{b}) \Rightarrow(\mathrm{c})$ : Let $F$ be any closed subset of $X$. Then $\mathrm{Cl}(F)=F$ and so $(\mathrm{b})$ implies that $\mathrm{Cl}\left[f^{\#}(F)\right] \subset f^{\#}(F)$. Hence $f^{\#}(F)$ is closed, and (c) holds.

(c) $\Rightarrow(\mathrm{a})$ : Let $U$ be any open subset of $X$. Then (c) implies that $f^{\#}\left(U^{C}\right)$ is closed in $Y$. But $f^{\#}\left(U^{C}\right)=[f(U)]^{C}$, by Lemma 2.3(vii). Therefore, $f(U)$ is open in $Y$. Hence $f$ is an open map. This proves (a).

Corollary 2.8. Let $f: X \rightarrow Y$ be onto. Then $f$ is an open map if and only if for each closed subset $F$ of $X$, $f\left(F^{\#}\right)$ is closed in $Y$.

Proof. The proof follows immediately from the equivalence of (a) and (c) in the above theorem and Lemma $2.5(\mathrm{~b})$.

In the next corollary, we see that interestingly images of saturated closed sets under open surjections are closed sets.

Corollary 2.9. Let $f: X \rightarrow Y$ be an open onto map. Then for each saturated closed subset $F$ of $X, f(F)$ is closed in $Y$. In particular, for any set $A$, if $A^{\#}$ is closed, then $f\left(A^{\#}\right)$ is closed in $Y$.

Theorem 2.10. For any map $f: X \rightarrow Y$, the following conditions are equivalent.

(a) $f$ is closed, that is, for each subset $A$ of $X, \mathrm{Cl}(f(A)) \subset f(\mathrm{Cl}(A))$;

(b) for each subset $A$ of $X, f^{\#}\left(A^{O}\right) \subset\left[f^{\#}(A)\right]^{O}$;

(c) for each open subset $U$ of $X, f^{\#}(U)$ is open in $Y$. 
Proof. (a) $\Rightarrow$ (b): By Lemma 2.3(vii) and condition (a), $f^{\#}\left(A^{O}\right)=\left[f\left(\left(A^{O}\right)^{C}\right)\right]^{C}=\left[f\left(\mathrm{Cl}\left(A^{C}\right)\right)\right]^{C} \subset$ $\left[\mathrm{Cl}\left(f\left(A^{C}\right)\right]^{C}=\left[\mathrm{Cl}\left(f^{\#}(A)\right)^{C}\right]^{C}=\left[f^{\#}(A)\right]^{O}\right.$. Hence, $f^{\#}\left(A^{O}\right) \subset\left[f^{\#}(A)\right]^{O}$, and $(\mathrm{b})$ holds.

(b) $\Rightarrow$ (c): If $U$ is any open subset of $X$, then $U=U^{O}$ and (b) implies that $f^{\#}(U) \subset\left[f^{\#}(U)\right]^{O}$. Therefore, $f^{\#}(\mathrm{U})$ is an open set and (c) holds.

(c) $\Rightarrow\left(\right.$ a): Let $F$ be any closed subset of $X$. Then (c) implies $f^{\#}\left(F^{C}\right)$ is open in $Y$. Therefore, part (vii) of Lemma 2.3 implies that $[f(F)]^{C}=f^{\#}\left(F^{C}\right.$ ) is open in $Y$. Thus, $f(F)$ is closed in $Y$, and hence $f$ is a closed map.

Corollary 2.11. Let $f: X \rightarrow Y$ be onto. Then $f$ is a closed map if and only if for each open subset $U$ of $X$, $f\left(U^{\#}\right)$ is open in $Y$.

Proof. The proof follows immediately from the equivalence of (a) and (c) in the above theorem, since $f\left(U^{\#}\right)=f^{\#}(U)$ if $f$ is onto by Lemma $2.5(\mathrm{~b})$.

In the next corollary we see that interestingly images of saturated open sets under closed surjections are open sets.

Corollary 2.12. Let $f: X \rightarrow Y$ be a closed onto map. Then for each saturated open set $U$ in $X, f(U)$ is open in $Y$. In particular, for any set $A$, if $A^{\#}$ is open, then $f\left(A^{\#}\right)$ is open in $Y$.

The remaining results of this section give characterizations of continuous maps.

The following Theorem 2.13 is an analog, in terms of interiors, of the result that a map $f: X \rightarrow Y$ is continuous if and only if for each subset $A$ of $X, f(\mathrm{Cl}(A)) \subset \mathrm{Cl}(f(A))$.

Theorem 2.13. A map $f: X \rightarrow Y$ is continuous if and only if for each subset $A$ of $X,\left[f^{\#}(A)\right]^{O} \subset f^{\#}\left(A^{O}\right)$.

Proof. We know that $\mathrm{f}$ is continuous if and only if for each subset $A$ of $X, f[\mathrm{Cl}(A)] \subset \mathrm{Cl}(f(A))$. But by part (vii) of Lemma 2.3, $f[\mathrm{Cl}(A)] \subset \mathrm{Cl}(f(A))$ if and only if $\left[f^{\#}\left((\mathrm{Cl}(A))^{C}\right)\right]^{C} \subset \mathrm{Cl}\left[\left(f^{\#}\left(A^{C}\right)\right)^{C}\right]$ $=\left[\left(f^{\#}\left(A^{C}\right)\right)^{O}\right]^{C}$. Therefore, $f[\mathrm{Cl}(A)] \subset \mathrm{Cl}(f(A))$ if and only if $\left[f^{\#}\left(A^{C}\right)\right]^{O} \subset f^{\#}\left(\left(A^{C}\right)^{O}\right)$. Since this equivalence holds for arbitrary subsets $A$ of $X$, we have $f$ is continuous if and only if $\left[f^{\#}(A)\right]^{O}$ $\subset f^{\#}\left(A^{O}\right)$ for each subset $A$ of $X$.

The following theorem characterizes continuity for onto maps.

Theorem 2.14. Let $f: X \rightarrow Y$ be any onto map. Then the following conditions are equivalent.

(a) $f$ is continuous;

(b) $\left[f\left(A^{\#}\right)\right]^{O} \subset f\left[\left(A^{O}\right)^{\#}\right]$ for all subsets $A$ of $X$;

(c) $A^{\#}$ is open in $X$ whenever $f\left(A^{\#}\right)$ is open in $Y$;

(d) for any saturated set $E$ in $X, E$ is open in $X$ whenever $f(E)$ is open in $Y$;

(e) for any saturated set $E$ in $X, E$ is closed in $X$ whenever $f(E)$ is closed in $Y$.

Proof. Since $(\mathrm{a}) \Leftrightarrow(\mathrm{b})$ by Theorem 2.13 above and Lemma $2.5(\mathrm{~b})$, we prove $(\mathrm{a}) \Rightarrow(\mathrm{c}) \Rightarrow(\mathrm{d}) \Rightarrow(\mathrm{e}) \Rightarrow$ (a).

(a) $\Rightarrow$ (c). Since $f^{\#}(A)=f\left(A^{\#}\right)$, by Lemma 2.5(b), (c) follows from (a) by Remark 1.1.

(c) $\Rightarrow$ (d). Since $E$ is saturated if and only if $E=E^{\#}$, (d) follows from (c) for arbitrary $f$.

$(\mathrm{d}) \Rightarrow(\mathrm{e})$. Let $f(E)$ be closed in $Y$, where $E$ is saturated in $X$. Then $[f(E)]^{C}$ is open in $Y$ and

$[f(E)]^{C}=f^{\#}\left(E^{C}\right)$, by Lemma 2.3(vii). Then $f$ is onto implies $[f(E)]^{C}=f\left[\left(E^{C}\right)^{\#}\right]$ by Lemma $2.5($ b). 
Also $E$ is saturated implies $E^{C}$ is also saturated and so $\left(E^{C}\right)^{\#}=E^{C}$, by Lemma 2.3(ix). Therefore, $[f(E)]^{C}=f\left(E^{C}\right)$ and so $f(E)$ closed in $Y$ implies that $f\left(E^{C}\right)$ is open in $Y$ and therefore, by $(\mathrm{d}), E^{C}$ is open and so $E$ is closed in $X$, proving thereby that (e) holds.

(e) $\Rightarrow\left(\right.$ a). Let $S$ be any closed subset of $Y$ and let $E=f^{-1}(S)$. Then $f(E)=S$, since $f$ is onto, and so $f(E)$ is closed for a saturated subset $E$ of $X$. Therefore, by condition (e), $E=f^{-1}(S)$ is closed in $X$. Hence, $f$ is continuous.

Corollary 2.15. A map $f: X \rightarrow Y$ is continuous if and only if for any saturated set $E$ in $X, E$ is open (closed) in $X$, whenever $f(E)$ is open (closed) in $f(X)$.

Proof. The proof follows from the equivalence of (a) and (d) ((a) and (e)) above since the map $f: X \rightarrow f(X)$ is onto.

\section{References}

[1] J. Dugundji, Topology, Prentice Hall, New Delhi, India, 1975.

[2] I. E. Schochetman, "A characterization of open mapping in terms of convergent sequences," International Journal of Mathematics and Mathematical Sciences, vol. 2006, Article ID 76162, 5 pages, 2006.

[3] A. V. Arkhangel'skii and V. I. Ponomarev, Fundamentals of General Topology: Problems E Exercises, Mathematics and Its Applications, Hindustan Publishing Corporation, New Delhi, India, 1984. 


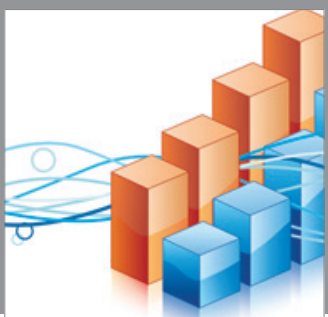

Advances in

Operations Research

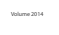

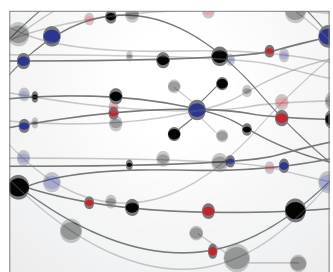

\section{The Scientific} World Journal
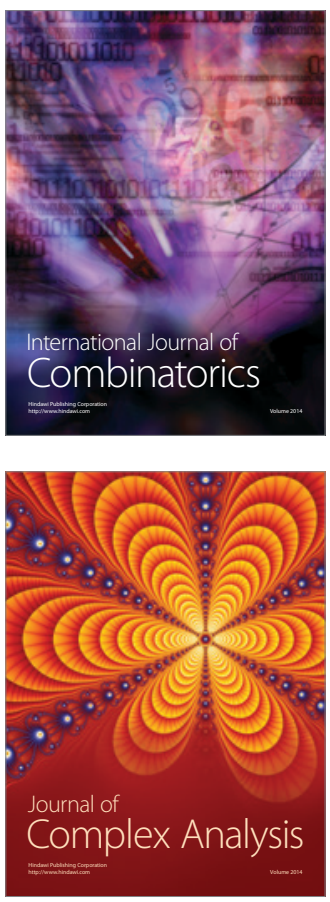

International Journal of

Mathematics and

Mathematical

Sciences
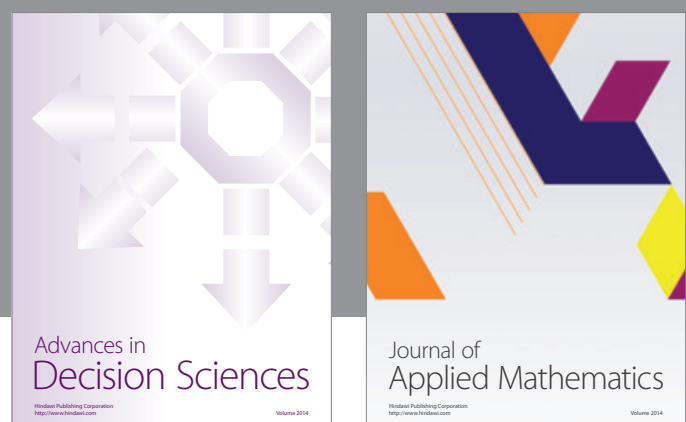

Journal of

Applied Mathematics
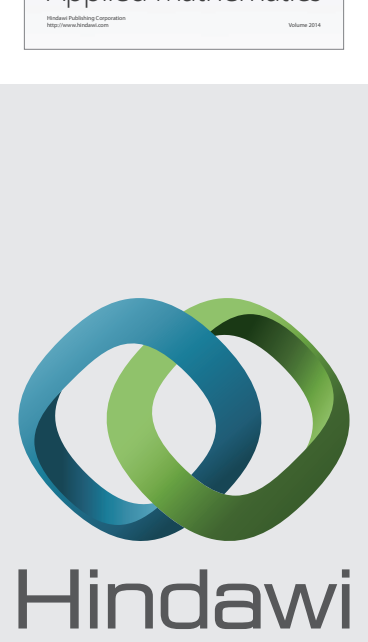

Submit your manuscripts at http://www.hindawi.com
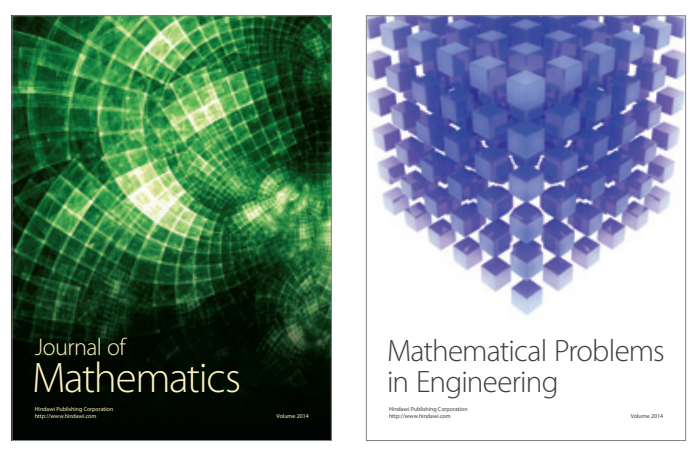

Mathematical Problems in Engineering
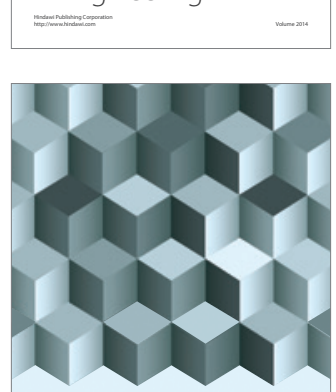

Journal of

Function Spaces
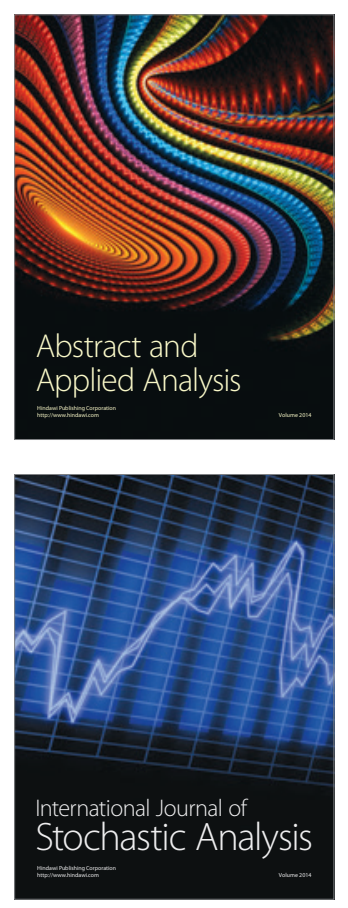

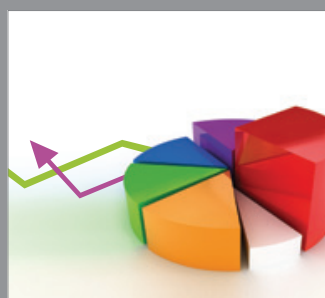

ournal of

Probability and Statistics

Promensencen
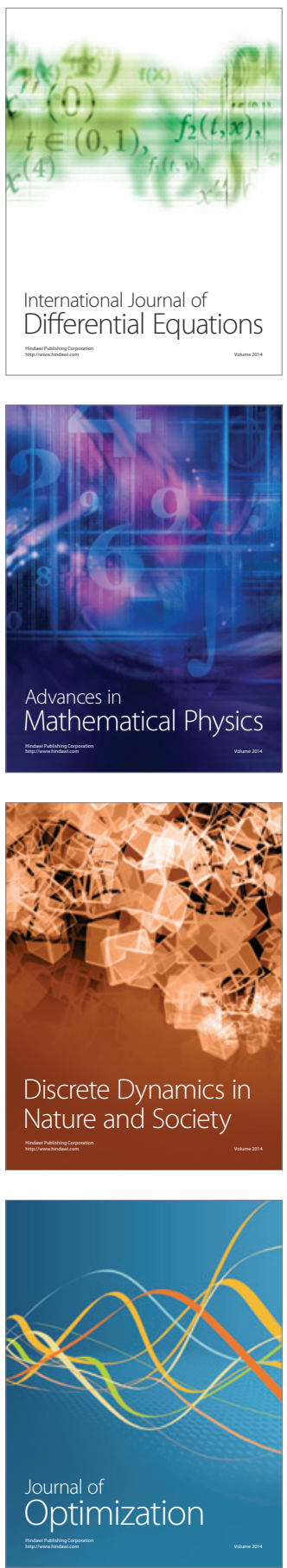